\title{
Prediction of CpG-island function: CpG clustering vs. sliding-window methods
}

\author{
Michael Hackenberg*1,2, Guillermo Barturen 1,2, Pedro Carpena3,4, Pedro L Luque-Escamilla5, Christopher Previti ${ }^{6}$ and \\ José L Oliver*1,2
}

\begin{abstract}
Background: Unmethylated stretches of $\mathrm{CpG}$ dinucleotides (CpG islands) are an outstanding property of mammal genomes. Conventionally, these regions are detected by sliding window approaches using \%G + C, CpG observed/ expected ratio and length thresholds as main parameters. Recently, clustering methods directly detect clusters of CpG dinucleotides as a statistical property of the genome sequence.

Results: We compare sliding-window to clustering (i.e. CpGcluster) predictions by applying new ways to detect putative functionality of $\mathrm{CpG}$ islands. Analyzing the co-localization with several genomic regions as a function of window size vs. statistical significance ( $p$-value), CpGcluster shows a higher overlap with promoter regions and highly conserved elements, at the same time showing less overlap with Alu retrotransposons. The major difference in the prediction was found for short islands (CpG islets), often exclusively predicted by CpGcluster. Many of these islets seem to be functional, as they are unmethylated, highly conserved and/or located within the promoter region. Finally, we show that window-based islands can spuriously overlap several, differentially regulated promoters as well as different methylation domains, which might indicate a wrong merge of several CpG islands into a single, very long island. The shorter CpGcluster islands seem to be much more specific when concerning the overlap with alternative transcription start sites or the detection of homogenous methylation domains.

Conclusions: The main difference between sliding-window approaches and clustering methods is the length of the predicted islands. Short islands, often differentially methylated, are almost exclusively predicted by CpGcluster. This suggests that $C p G c l u s t e r$ may be the algorithm of choice to explore the function of these short, but putatively functional $\mathrm{CpG}$ islands.
\end{abstract}

\section{Background}

The methylation of $\mathrm{CpG}$ dinucleotides is an important epigenetic modification of DNA, required in mammals for embryonic development, genomic imprinting and $\mathrm{X}$ chromosome inactivation [1-3]. Around $80 \%$ of all CpG dinucleotides are methylated in mammal genomes. The exceptions are short stretches of CpG dinucleotides (CpG islands or CGIs), which are predominantly hypomethylated in healthy tissues $[4,5]$. CGIs are thought to be predominantly located in the promoter region of genes; around $70 \%$ of all genes have a CGI overlapping its pro-

*Correspondence: mlhack@gmail.com, oliver@ugr.es ${ }^{1}$

Dpto. de Genética, Facultad de Ciencias, Universidad de Granada, Campus de Fuentenueva s/n, 18071, Granada, Spain

1 Dpto. de Genética, Facultad de Ciencias, Universidad de Granada, Campus de Fuentenueva s/n, 18071, Granada, Spain

Full list of author information is available at the end of the article moter region. Moreover, virtually all housekeeping genes are associated to CGIs, while only half of the tissue specific genes show such association [6]. Given its location in the promoters, CGIs may play important roles in the regulation of gene expression. An example is the aberrant methylation of CGIs observed in many cancer types [711]. Moreover, evidence exist that the differential or tissue specific methylation of CpG islands may be involved in the regulation of tissue specific genes [12].

Accurate prediction tools are therefore needed and a considerable effort has been carried out over the last decade to detect CGIs in mammal genomes. Many different algorithms have been proposed, most of them based on the criteria of Gardiner-Frommer [1]. These authors proposed in 1987 thresholds for the detection of CGIs: GC-content (50\%), CpG observed/expected (O/E) ratio (0.6) and length (200 bp). Many of the published methods

@ 2010 Hackenberg et al; licensee BioMed Central Ltd. This is an Open Access article distributed under the terms of the Creative ComBHWed Central mons Attribution License (http://creativecommons.org/licenses/by/2.0), which permits unrestricted use, distribution, and reproduction in any medium, provided the original work is properly cited. 
simply readjust these thresholds. However, it has been shown that filtering criteria-based definitions of $\mathrm{CpG}$ islands are mathematically incomplete and non-operational, as the sliding window methods frequently fail to identify a large percentage of subsequences that meet the filtering criteria [13].

Recently, methods based on the clustering of CpGs along the genome sequence detect CGIs as a statistical property, thereby not relying on thresholds of GC-content, $\mathrm{O} / \mathrm{E}$ ratio and length. The first algorithm published in this category was the CpGcluster method [14], which detects the CGIs by means of the distances between CpGs, then assigning a statistical significance to each cluster of CpG dinucleotides. Subsequently, Cp Gcluster was followed by other methods detecting CGIs by means of the CpG densities [15-18]. In the same way, many other features could also contribute to determine the boundary of individual CpG-islands, such as transcription factors and nucleosome location. The nucleosome code could be an important ingredient of future CGI models, although sequence features will probably remain as the principal component (see, for example, [19]). Epigenetic information may be also of help in detecting CGIs by making use of contextual information [20].

Given the conceptual differences between sliding window algorithms (SWA) using a high parameter space and those detecting CGIs as a statistical property of the CpG clustering in DNA sequences, disagreement exist on the way CGIs should be predicted. Recently, a comparison between islands detected by the window-based TakaiJones (TJ) program [21] and those detected by CpGcluster was published [22]. The comparison evaluated mainly the co-localization of CGIs and known promoters and concludes an overall advantage for the TJ approach over CpGcluster.

We present here new ways to detect putative function of CGIs, emphasizing the basic difference between $C p G$ cluster and SWA predictions: the statistical significance introduced by $C p G c l u s t e r$ instead of the conventional length threshold. We show that the statistical significance assigned to each $C p G c l u s t e r$ island is a key criterion to control the overlap with promoter regions, evolutionarily conserved elements and spurious Alu elements. Finally, we show that many short $(<200 \mathrm{bp}$ ) islands (CpG islets) may be also functional, given its overlap with either promoter or evolutionary conserved regions and the absence of methylation in at least one tissue. As many of these islets are exclusively predicted by $C p G$ cluster, this may be the algorithm of choice for experimental essays aimed to verify the function of these short islands.

\section{Results and Discussion}

The way sliding-window approaches and CpGcluster detect CGIs are conceptually different. While SWA detect regions above the thresholds of $\mathrm{G}+\mathrm{C}, \mathrm{O} / \mathrm{E}$, min $\mathrm{CpG}$ and length, CpGcluster predicts statistically significant clusters of CpGs as CGIs. As a first consequence, the statistical properties of the predicted islands are different as well (Figure 1); e.g. in SWA approaches the distributions of important CGI properties like $\% G+C$ and $O / E$ ratio are heavily biased towards the user thresholds.

Therefore, the first part of this work is basically aimed to clarify: 1) the differences between the length threshold used by SWA and the statistically significance used by CpGcluster; and 2) the consequences that the differences in the number of predicted islands and the mean length might have on the prediction quality.

Prediction quality has been assigned conventionally by the percentage of overlap with promoter regions and spurious $A l u$ elements. In the original publication of $C p G$ cluster [14] we added the overlap with evolutionarily conserved elements or PhastCons [23] as an indicator of putative functionality. Here, we add several new types of analysis to assess the prediction quality, namely the capability to distinguish between different methylation domains or different alternative Transcription Start Sites (TSSs).

\section{CpG islands in the promoter region}

Since CpG islands are preferentially located in the promoter region of genes, this fact has been extensively used to assess the quality of CGI predictions [24]. Recently, it has been claimed [22] that a higher percentage of TJ islands (35\%) are located within the promoter when compared to CpGcluster islands (14.7\%). In Table 1, we show a similar analysis as carried out in [22], but extending the comparison to other window based programs and different prediction sets for the CpGcluster algorithm. When considering $C p$ Gcluster islands with $p$-value $\leq 1 \mathrm{E}-5$ (the original relaxed set), the CGI fraction overlapping the promoter region is effectively smaller than for the other programs. However, note that the numbers of CGIs predicted by window-based methods are far below the number predicted by $C p G$ cluster. To allow for an unbiased comparison, we obtained a second, strict set of CpGcluster islands simply by increasing the required statistical significance to $p$-value $\leq 1 \mathrm{E}-20$ (i.e. filtering out the less significant islands), then obtaining a total 25,454 CGIs. This number is within the range of recent estimates for the complete human somatic cell CGI complement [25]. The strict, more statistically significant set of CpGcluster islands shows now the highest overlap (52.4\%) with the promoter region. This advantage looks even more important when considering that the genome coverage of our strict set $(0.65 \%)$ was the lowest one. This indicates a high specificity of CpGcluster, which strongly supports our original claim that the $p$-value is the most important 

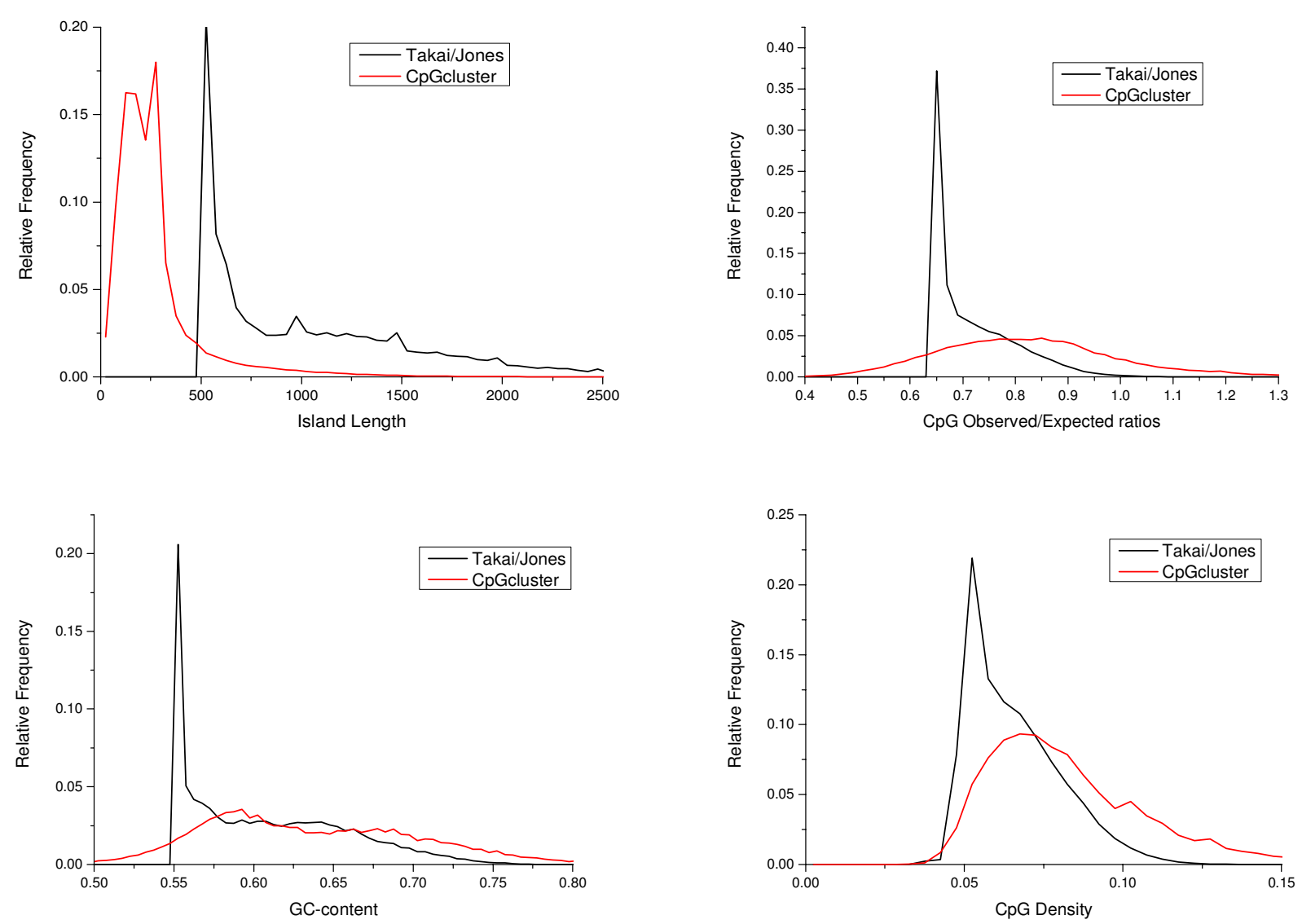

Figure 1 Comparison of the distributions of the island length for both the CpGcluster and Takai/Jones algorithm (top left); the observed to expected ratios of CpG frequencies (top right); the island GC-content (bottom left); and the island CpG density (bottom right). It can be seen that, for all this four properties, the SWA distributions are heavily biased towards their respective thresholds. However, C $p$ Gcluster distributions do not show this artifact.

parameter to distinguish promoter CGIs from the rest of genome islands [14].

\section{A comparison of length and $p$-value thresholds}

The main quality parameter in SWA is the window size (CGI length threshold). Originally, the window size was set to $200 \mathrm{bp}$ to assure that the detected regions surpass the $\mathrm{G}+\mathrm{C}$ and $\mathrm{O} / \mathrm{E}$ criterion not due to chance alone [1] Subsequently, this threshold was increased to $500 \mathrm{bp}$ in order to reduce the false positive rate by eliminating spurious Alu elements [21]. This criterion was replaced in $C p$ Gcluster by the statistical significance ( $p$-value), a more robust and reliable way to distinguish true CGIs from stochastic noise, disregard island length [14]. Note that the $p$-value is not just a different expression for the island length. A non-linear relation exists between the $p$ values and the lengths of the predicted CpGcluster islands, as the $p$-value depends on both the island length and the island density (Figure 2).
To evaluate the discrimination power of CpGcluster $p$ value against window size, we generated a series of island-set predictions, each one containing the same number of islands, by appropriately varying the window size or the $p$-value thresholds. Next, we determined the overlap of the resulting islands with the promoter regions, PhastCons elements [23] and Alu repeats. The island sets selected by $p$-value clearly outperformed those selected by length: a higher percentage of CpGcluster islands overlap with promoters (Figure 3) and PhastCons elements (Figure 4) along the entire range of the two parameters, at the same time reducing the overlap with spurious Alu elements (Figure 5). Table 2 shows the correspondence between the number of predicted islands, $p$ value and window length.

The results in Figures 3, 4, 5 are straightforward in comparing the relative strengths of the two main parameters involved in CGI quality (length and p-value). The increased stringency in the conventional parameters used by the TJ program excluded contaminating Alu elements, 
Table 1: Co-localization of CpG islands and the promoter region.

\begin{tabular}{|c|c|c|c|c|}
\hline \multirow[t]{2}{*}{ Method } & \multirow[t]{2}{*}{ Number of predicted islands } & \multirow[t]{2}{*}{ Genome coverage (\%) } & \multicolumn{2}{|c|}{ Promoter overlap (R13) } \\
\hline & & & Number of islands & $\%$ \\
\hline $\mathrm{TJ}$ & 37,323 & 1.43 & 14,034 & 37.60 \\
\hline UCSC & 27,639 & 0.74 & 13,369 & 48.40 \\
\hline CpGproD & 76,886 & 2.81 & 14,814 & 19.30 \\
\hline \multicolumn{5}{|l|}{ CpGcluster: } \\
\hline relaxed set* & 198,702 & 1.90 & 30,660 & 15.43 \\
\hline strict set ${ }^{* *}$ & 25,454 & 0.65 & 13,349 & 52.40 \\
\hline
\end{tabular}

but it also reduced the number of gene promoter associated islands, suggesting that bona fide CGIs were also being discarded [25]. However, raising the statistical significance (i.e. decreasing the p-value) of CpGcluster leads to an exponential increase in the overlap with promoters or PhastCons, simultaneously decreasing the overlap with Alu elements. CpGcluster algorithm is, therefore, a more rational and powerful way to increase CGI prediction quality. An additional advantage is that CpGcluster p-value would be particularly useful in comparative genomics of CGIs, making possible the comparison of CGIs with the same statistical significance, but obtained from different species, despite variations in $\mathrm{G}+\mathrm{C}$ content or CpG density.

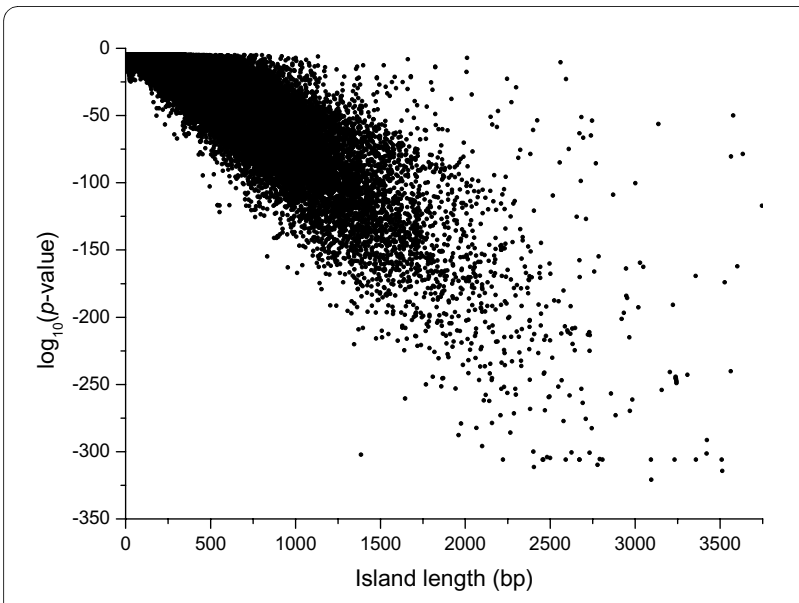

Figure 2 The length of $C p G c l u s t e r$ islands vs. the logarithm of the assigned $\boldsymbol{p}$-value. It can be seen that no linear correlation exists and that the relation between $p$-value and length is more complex, e.g. the $p$-value depends on both the island length and the island density.

\section{Prediction of unmethylated regions}

The most important criterion to assess putative functionality of a CpG island is the absence of methylation. Therefore, the comparison to experimentally verified, unmethylated regions is another important analysis type to establish prediction quality.

Recently, the methylation status of 697 hypermethylated and 6,987 hypomethylated promoter regions in WI38 primary lung fibroblast [26] have been used to compare the prediction quality of $\mathrm{TJ}$ and $C p$ Gcluster algorithms [22]. In this study, the prediction quality was measured in the following way: i) true positives (TP): hypomethylated promoters containing a predicted island, ii) false positives (FP): hypermethylated promoters containing a predicted island, iii) true negatives (TN): hypermethylated promoters not containing a predicted island, and iv) false negatives (FN): hypomethylated promoters not containing a predicted island.

However, in our opinion, there is an important pitfall in such an approach. It is known that the methylation state of a given region can change among different tissues; therefore, assigning a "false positive" label to a predicted island which has been shown to be methylated in a single tissue may be misleading, as the same prediction could be perfectly "true positive" if measured in a different tissue.

Fortunately, Weber et al. [26] also determined the methylation states in sperm. Analyzing fibroblast and sperm data together, we observed that 11,260 regions are unmethylated in both tissues but 1,550 are unmethylated in one tissue but methylated in the other one. This means that around $12 \%$ of the regions are differentially methylated; therefore, a substantial number of FPs were actually TPs. Given these data, in our opinion, without the knowl- 
Table 2: Correspondence between the number of predicted islands, log ( $p$-value) and window length.

\begin{tabular}{lcc}
\hline No. of predicted islands & log (p-value) & Window length \\
\hline 193,856 & 5.06509 & 200 \\
\hline 139,013 & 6.1864 & 250 \\
\hline 109,907 & 7.19943 & 300 \\
\hline 69,477 & 9.82744 & 350 \\
\hline 52,687 & 11.85626 & 400 \\
\hline 42,392 & 13.73824 & 450 \\
\hline 37,293 & 14.96788 & 500 \\
\hline 33,691 & 15.95388 & 500 \\
\hline 30,881 & 16.8824 & 600 \\
\hline 28,162 & & \\
\hline 26,192 & 18.18919 & \\
\hline & & \\
\hline & & \\
\hline & & \\
\hline
\end{tabular}

edge of the methylation state in a vast number of different tissues, the number of "false positive predictions" cannot be assessed in this way.

We therefore based our quality assessment on sensitivity, a measure not dependent on the false positive rate, as well as on the estimation of the lower bound for the posi-

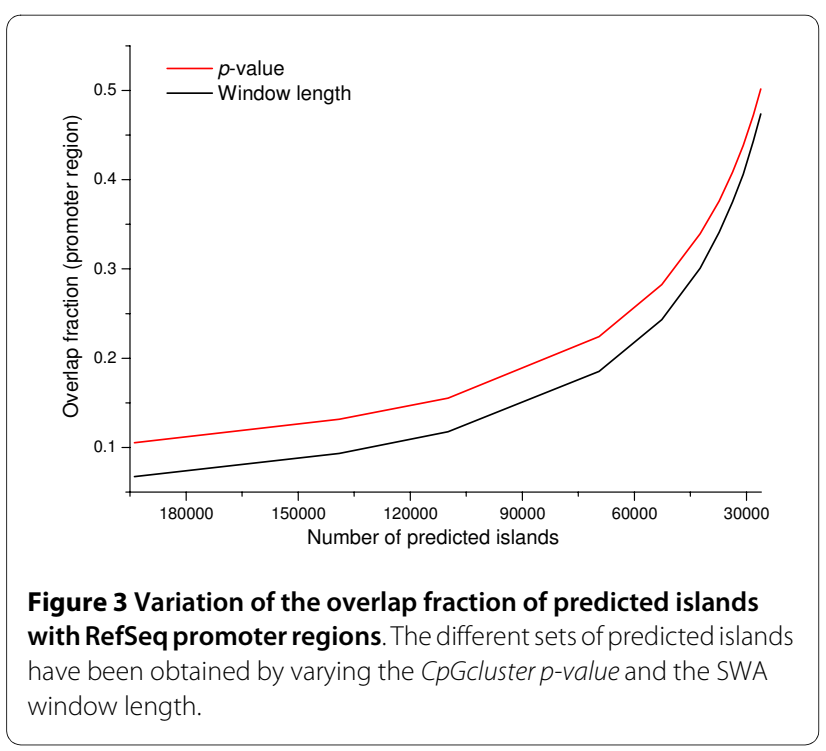

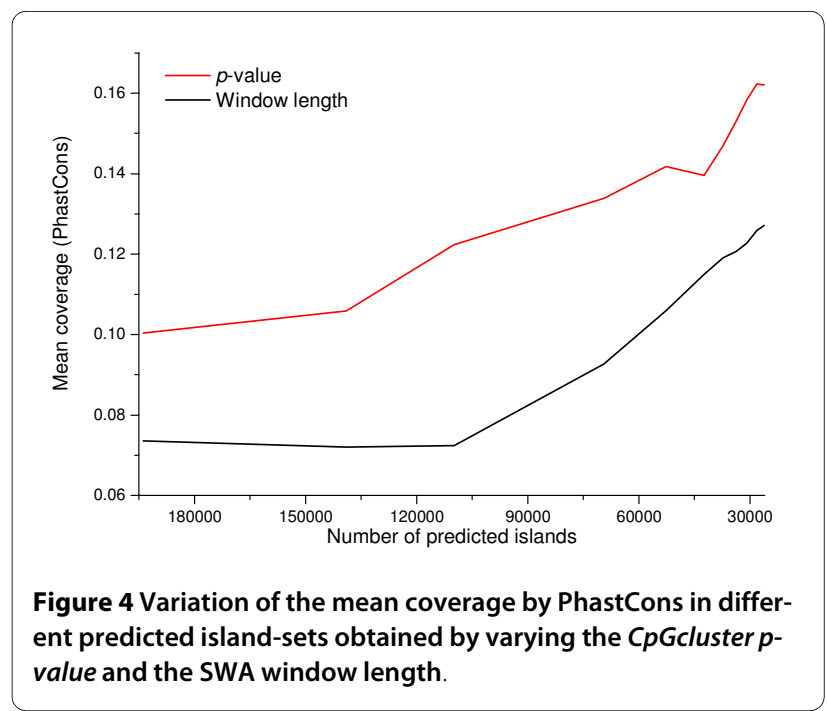

tive predictive value (PPV, see Data and Methods), a measure used in the gene prediction field under the name of specificity [27]. We used two different experimentally validated sets of unmethylated regions (see Data and Methods) to assess the quality of the 5 sets of predicted islands. Table 3 depicts the results when taking genome-wide, experimentally verified unmethylated $\mathrm{CpG}$ islands as reference (Bird's islands, [28]). The table shows that the Cp Gcluster relaxed set shows the highest sensitivity while the strict set shows the lowest one. When considering the lower boundary of the PPV (i.e. the method is at least as specific as this value), we observed the contrary pattern, the CpGcluster strict set now shows the highest PPV, while the relaxed set shows the lowest one. Table 4 seems to confirm this trend when using unmethylated regions which are mainly related to promoters [26]. These results indicate that CpGcluster is either the most sensitive or the most specific algorithm, depending on the applied $p$ -

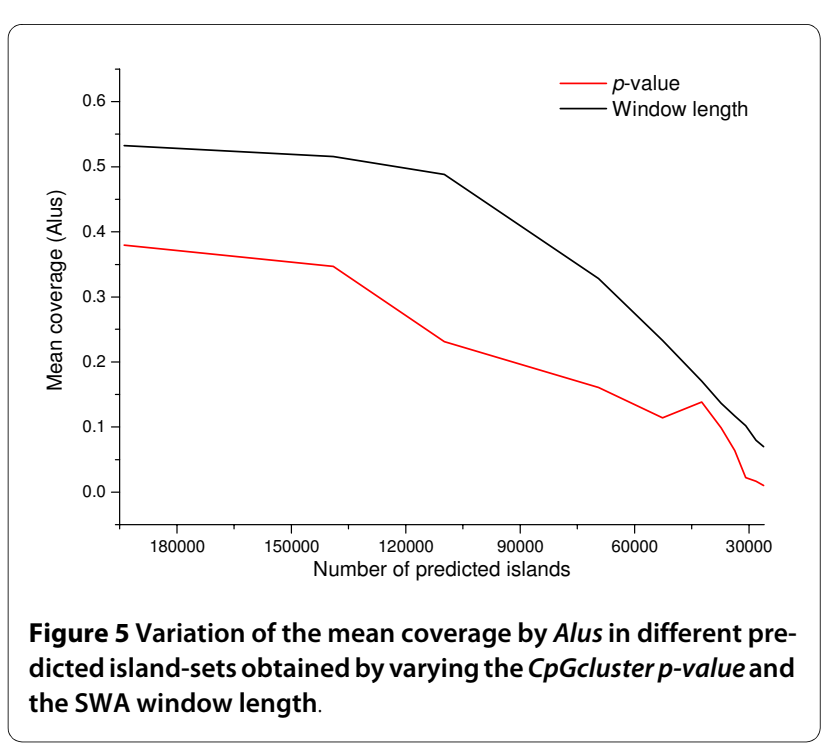


Table 3: Prediction of unmethylated regions (Bird's islands, $\mathrm{N}=17,383$ ).

\begin{tabular}{|c|c|c|c|c|c|}
\hline Method & $\begin{array}{l}\text { Number of predicted } \\
\text { islands }\end{array}$ & $\begin{array}{l}\text { Number of islands } \\
\text { overlapping a Bird's island }\end{array}$ & $\begin{array}{l}\text { Number of Bird's islands } \\
\text { 'touched' by the prediction }\end{array}$ & SN & PPV \\
\hline TJ & 37,293 & 14,315 & 14,942 & 0.854 & 0.384 \\
\hline UCSC & 27,639 & 13,858 & 14,256 & 0.816 & 0.501 \\
\hline CpGproD & 76,886 & 14,250 & 15,346 & 0.875 & 0.185 \\
\hline \multicolumn{6}{|l|}{ CpGcluster: } \\
\hline relaxed set* & 198,702 & 29,235 & 15,497 & 0.939 & 0.147 \\
\hline strict set ${ }^{* *}$ & 25,454 & 14,809 & 12,623 & 0.757 & 0.582 \\
\hline
\end{tabular}

value threshold. The finding for the relaxed set confirms the result reported by Han and Zhao [22]. Note, however, that $C p G c l u s t e r$ strict set reaches the highest specificity but the lowest sensitivity. Interestingly, a recent study [29] also emphasizes that the CpGcluster p-value is a key attribute for distinguishing between constitutively methylated and unmethylated CGIs.

\section{CpG islands in the domains bound by polycomb repressive} complex 2

Functional clusters of CpGs are not limited to promoter regions, they are also found in other genomic locations. An example are the hyperconserved CpG domains largely overlapping the domains bound by polycomb repressive complex 2 (PRC2) [30], located far from the promoter and playing an important role in transcriptional silencing during development. We determined the overlap of the CGIs predicted by different finders with the domains bound by PRC2. Table 5 shows that all the finders show high sensitivities and low PPVs in predicting these sites, being CpGcluster the algorithm obtaining the highest sensitivity (relaxed set).

\section{Functional specificity vs. length of $\mathrm{CpG}$ islands}

One of the most striking differences between SWA and the CpGcluster approach is the length of the predicted islands. SWA islands are on average much longer than CpGcluster islands (TJ $=1,094.9$; UCSC $=764.5$; CpGProD = 1,046.1; CpGcluster $=273.2$ (relaxed set), or 727.5 (strict set)). Originally, CGIs were estimated to be on

Table 4: Prediction of unmethylated regions (Weber's regions, $N=13,277$ ).

\begin{tabular}{|c|c|c|c|c|c|}
\hline Method & $\begin{array}{l}\text { Number of } \\
\text { predicted } \\
\text { islands }\end{array}$ & $\begin{array}{c}\text { Number of } \\
\text { islands } \\
\text { overlapping a } \\
\text { Weber's region }\end{array}$ & $\begin{array}{c}\text { Number of } \\
\text { Weber's regions } \\
\text { 'touched' by the } \\
\text { prediction }\end{array}$ & SN & PPV \\
\hline TJ & 37,293 & 10,179 & 9,965 & 0.755 & 0.273 \\
\hline UCSC & 27,639 & 9,788 & 9,552 & 0.724 & 0.354 \\
\hline CpGproD & 76,886 & 10,320 & 10,257 & 0.774 & 0.134 \\
\hline \multicolumn{6}{|l|}{ CpGcluster: } \\
\hline relaxed set* & 198,702 & 18,967 & 10,372 & 0.867 & 0.095 \\
\hline strict set ${ }^{* *}$ & 25,454 & 9,633 & 8,378 & 0.663 & 0.378 \\
\hline
\end{tabular}

${ }^{*} p$-value $\leq 1 \mathrm{E}-5 ;{ }^{* *} p$-value $\leq 1 \mathrm{E}-20$ 
Table 5: Overlap of different CGIs with 3,465 domains bound by the polycomb repressive complex 2 (PRC2).

\begin{tabular}{|c|c|c|c|c|c|}
\hline Method & $\begin{array}{l}\text { Number of predicted } \\
\text { islands }\end{array}$ & $\begin{array}{c}\text { Number of islands } \\
\text { overlapping PRC2 domains }\end{array}$ & $\begin{array}{l}\text { Number of PRC2 domains } \\
\text { 'touched' by the prediction }\end{array}$ & SN & PPV \\
\hline TJ & 37,293 & 3,523 & 3,033 & 0.891 & 0.094 \\
\hline UCSC & 27,639 & 3,179 & 2,790 & 0.825 & 0.115 \\
\hline CpGproD & 76,886 & 3,321 & 3,159 & 0.916 & 0.043 \\
\hline \multicolumn{6}{|l|}{ CpGcluster: } \\
\hline relaxed set* & 198,702 & 9,097 & 3,097 & 0.961 & 0.046 \\
\hline strict set $^{* *}$ & 25,454 & 3,424 & 2,372 & 0.758 & 0.135 \\
\hline
\end{tabular}

average $1 \mathrm{~kb}$ long [1]. Frequently, more than one $C p G$ cluster island can be found within the promoter region and furthermore, several CpGcluster islands are often embedded within one single conventional, SWA island. For instance, around $53 \%$ of all $\mathrm{TJ}$ islands host more than one CpGcluster island (Figure 6).

Given these facts, it might be that either conventional SWA predictions erroneously merge smaller islands into longer ones, or that CpGcluster erroneously fragments longer islands into many smaller ones. Next, we use alternative TSSs and single CpG resolution methylation data to shed light on these questions.

\section{Alternative promoters}

Frequently, CpGcluster predicts more than one island within the promoter region. It has been shown [22] that $37.8 \%$ of all RefSeq genes have more than one CpGcluster island, while only $3.2 \%$ have more than one $\mathrm{TJ}$ island. Following the premise "one promoter one CpG island", this observation was interpreted as a disadvantage of $\mathrm{CpG}$ cluster [22]. However, in recent years, new insights into the regulation of gene expression became available, showing among other things a frequent use of alternative TSSs. The existence of alternative TSSs opens the possibility that more than one island per gene might exist. Therefore, the high percentage of genes with more than one Cp Gcluster island might instead indicate a more specific relation of $C p$ Gcluster islands to alternative promoters or TSSs. To check this possibility, we used the DBTSS database [31]. Out of 15,194 RefSeq genes annotated in the latest DBTSS release, 7,895 (52\%) have at least one alternative TSS. With such scenario, one might expect up to $52 \%$ of all promoters having more than one island in its promoter (one for each TSS). Given these numbers, the reported $37.8 \%$ of genes with more than one CpGcluster island might look not so inadequate.

Conversely, this finding might indicate that the $\mathrm{TJ}$ algorithm artificially joins several functional islands into one single longer island. To further investigate this possibility, we estimated the number of islands simultaneously overlapping multiple TSSs annotated in the DBTSS database. Table 6 shows that the CpGcluster sets, both relaxed and strict, overlap a higher fraction of unique, and a lower fraction of multiple TSSs than the islands predicted by other programs, thus making CpGcluster predictions much more specific in overlapping individual TSSs.

Figure 7 shows a particular example of a bidirectional promoter region. The TSSs of the two genes, UFD1L and CDC45L, are overlapped by the same TJ or UCSC island, while $C p$ Gcluster predicts separate islands. This is inter-

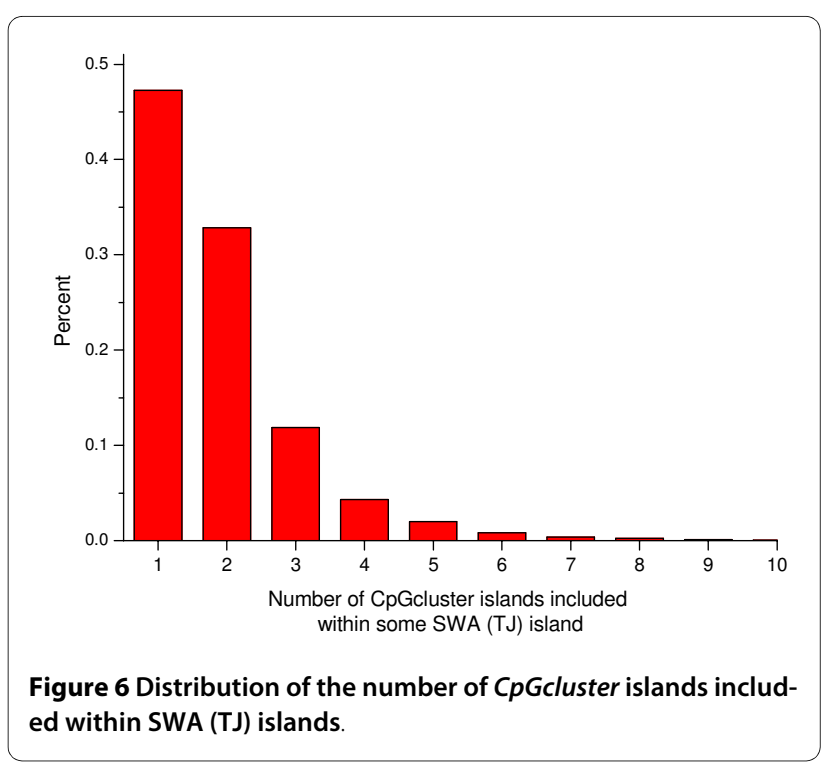


esting, as these two genes have very different expression breadths. Using the GeneAtlas2 expression data [32], we determined for UFD1L an expression breadth of $97.3 \%$ (expressed in 71 out of 73 healthy tissues), being therefore a housekeeping gene, while the CDC45L gene is expressed in just $15.1 \%$ (11 of 73) of all tissues. Given this differential gene expression pattern, a shared CpG island seems to be less specific than the scenario where each of the genes has its own island, as suggested by the prediction of CpGcluster.

In the human genome, there are a total of $166 \mathrm{bi}$-directional promoter pairs which share one long SWA CGI but two separated CpGcluster CGIs. The gene-pair shown in Figure 7 may be just an example of extreme differentiation in gene-expression: while the first member of the gene-pair is a housekeeping gene, the second one is a tissue-specific gene. However, one cannot reasonably expect that this may be the rule for all the bidirectional gene-pairs. In fact, after analyzing the expression profiles in a sample of 73 healthy tissues, only 16 (or 9.64\%) genepairs show a completely divergent pattern of gene-expression (coexpression value $\leq 0.2$, see Methods), while 13 (or 7.83\%) exhibit complete coexpression (coexpression value $=1$ ). The remaining gene-pairs show intermediate values of coexpression.

On the other hand, by using single base resolution methylation data [33], we also analyzed methylation differences between the CGIs overlapping bi-directional promoters. We found that 10 (or $11.24 \%$ ) of these islandpairs in $\mathrm{H} 1$ stem cells, and 15 (or 16.85\%) in the IMR90 fetal lung fibroblasts, show significant differences (MannWhitney non-parametric test) in their methylation average $(\mathrm{p} \leq 0.05)$.

\section{Heterogeneous methylation in long SWA islands}

A functional $\mathrm{CpG}$ island should show a rather homogenous methylation profile among the different $\mathrm{CpGs}$ and over the different tissues. For example, the existence of more than one methylation domain within a predicted island might indicate an erroneous merging of two small islands into a single longer island.

Here, we used single base resolution methylation data from different sources (see Data and Methods) to decide whether CpGcluster predicts too many short islands or SWA predict too many long islands. In doing so, we detect all TJ islands which harbor at least two CpGcluster islands. Next, we calculate the mean methylation for each Cp Gcluster island and the maximal difference in methylation over the different tissues. If many $\mathrm{TJ}$ islands exist with high methylation differences inside, this might indicate an erroneously joining of different methylation domains into a single island. Figure 8 shows a particular example from human chromosome 22. The region for which HEP data were available is just 317 bp long, showing a very pronounced change of the methylation values in embryonic liver cells. All SWA programs predict a very long island in this region, including completely the interesting region where the un-methylation/methylation border occurs. Only CpGcluster predicts precisely one CGI for each of the methylation domains.

Figure 9a shows the distribution of the maximum differences in the methylation of CpGs inside TJ islands for HEP data. It can be seen that very high differences occur, around $12 \%$ of all tested islands having higher differences than $30 \%$ in methylation. Methylation HEP data are available for only $5 \%$ of all tissues, and therefore the $12 \%$ of heterogeneous $\mathrm{TJ}$ islands merging several methylation domains might increase when data for more tissues

Table 6: Co-localization of CpG islands and alternative promoters.

Numbers of overlapping islands

\begin{tabular}{lccc}
\hline Method & All the TSSs & Unique TSS & Multiple TSSs \\
\hline TJ & 13,759 & $8,868(64.45 \%)$ & $4,891(35.55 \%)$ \\
\hline UCSC & 11,826 & $8,143(68.86 \%)$ & $5,518(31.14 \%)$ \\
\hline CpGproD & 15,319 & $9,801(63.98 \%)$ & $5,518(36.02 \%)$ \\
\hline CpGcluster islands: & & & $3,061(20.28 \%)$ \\
\hline relaxed set ${ }^{*}$ & 15,095 & $12,034(79.72 \%)$ & $2,666(25.82 \%)$ \\
\hline strict set ${ }^{* *}$ & 10,325 & $7,659(74.18 \%)$ & \\
\hline${ }^{*} p$-value $\leq 1 \mathrm{E}-5 ;{ }^{*} p$-value $\leq 1 \mathrm{E}-20$ & &
\end{tabular}




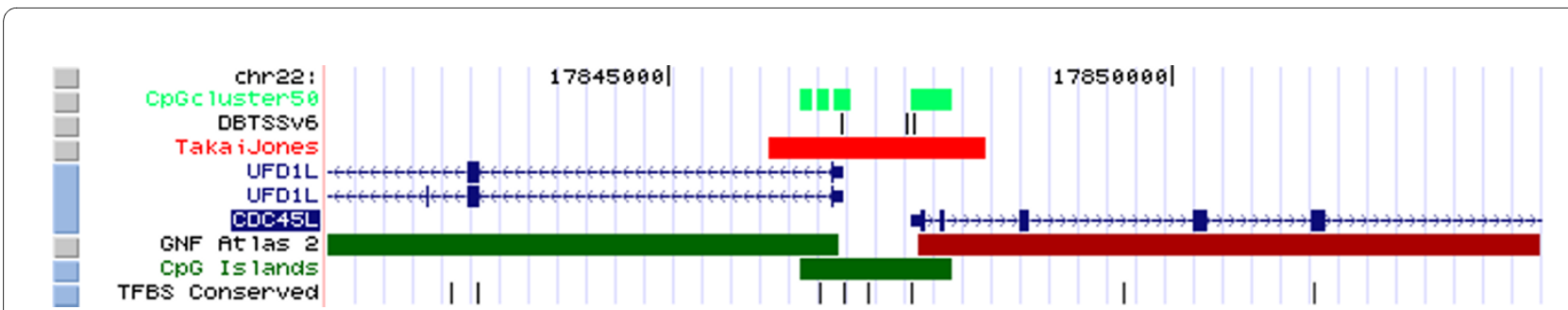

Figure 7 A bidirectional promoter region in human chromosome 22 which is overlapped by one TJ or UCSC island but by several CpGcluster islands. The two genes show very different expression profiles, and therefore it is very likely that the prediction of different islands for the different TSSs as done by CpGcluster is the better choice. The figure was obtained by using the UCSC Genome Browser [46]

becomes available. A similar conclusion can be reached when methylation data for two human methylomes [33] were used (Figure 9b). Note that the complex methylation structure within $\mathrm{CpG}$ islands has been reported before within a different context, but also showing that many long CpG islands contain more than one methylation domain [34].

\section{CpG-islets}

CpG "islets", genomic regions not conventionally classified as CGIs because of their short length $(<200 \mathrm{bp})$, but having a GC content and observed-to-expected CpG ratio characteristic of a CGI, have recently been identified in a 6.76-Mbp chromosomal region (10q25) containing a neocentromere [35]. Some of these islets remain unmethylated, corresponding to sites of active transcription and/ or boundaries that separate major chromatin subdomains. This suggests that, as conventional islands, the islets can also participate in the maintenance of a particular genomic pattern of methylated/unmethylated CpGs, thus contributing to the differential regulation of gene expression [3-5].

Given their tiny size, islets remain undetected by SWA, conventional CGI finders $[2,21,36-40]$, as all these programs share a length threshold above 200, or even $500 \mathrm{bp}$. Such length thresholds make conventional finders useless for the detection of $\mathrm{CpG}$ islets, since a relaxation of the length threshold will lead to a strong increment of false

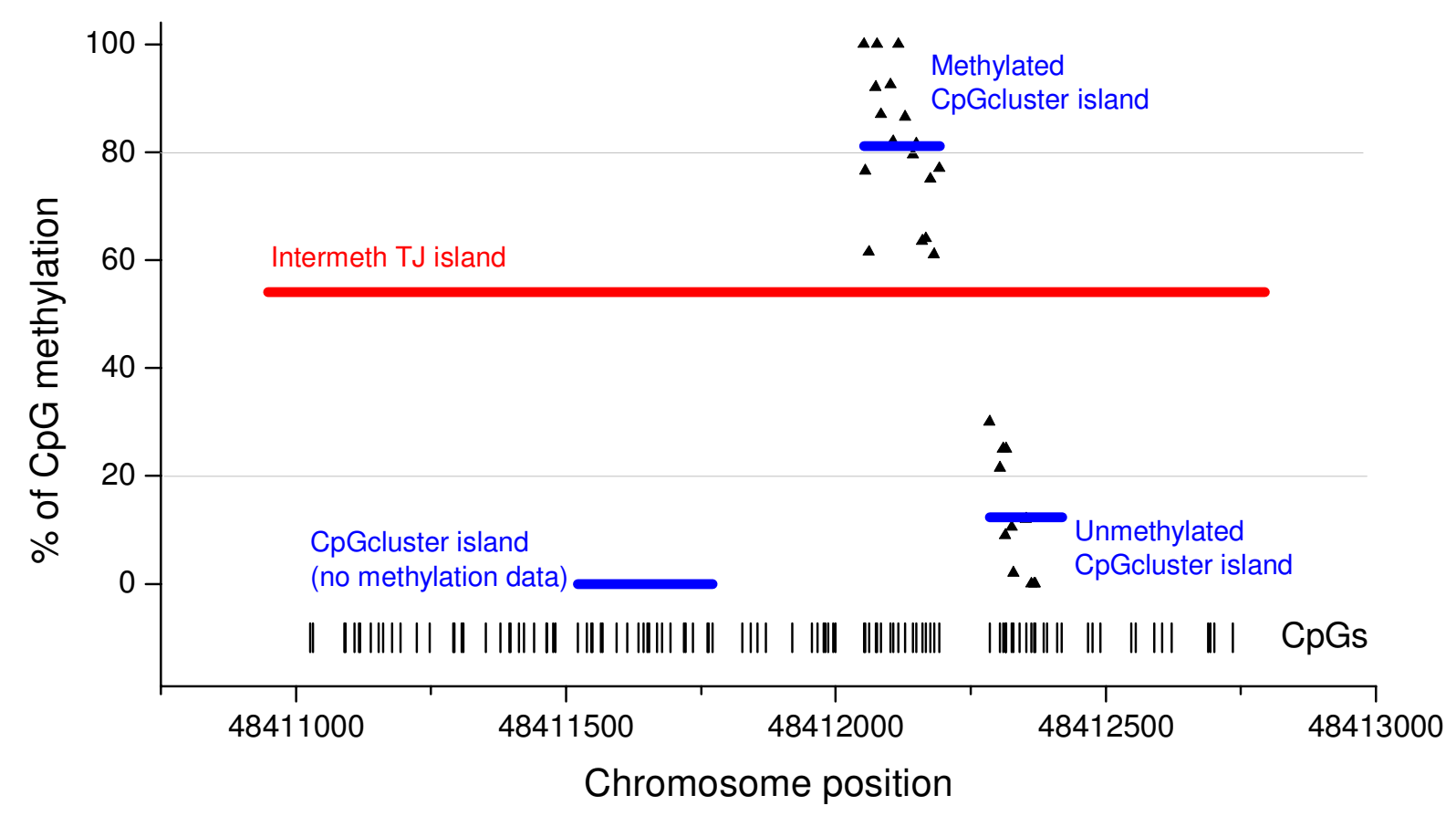

Figure 8 A 317 bp long region of human chromosome 22 showing strong heterogeneity in methylation. CpGcluster predicts separate islands for each methylation domain, while $\mathrm{TJ}$ and all the remaining tested sliding-window approaches predict only one longer island overlapping the different methylation domains. 

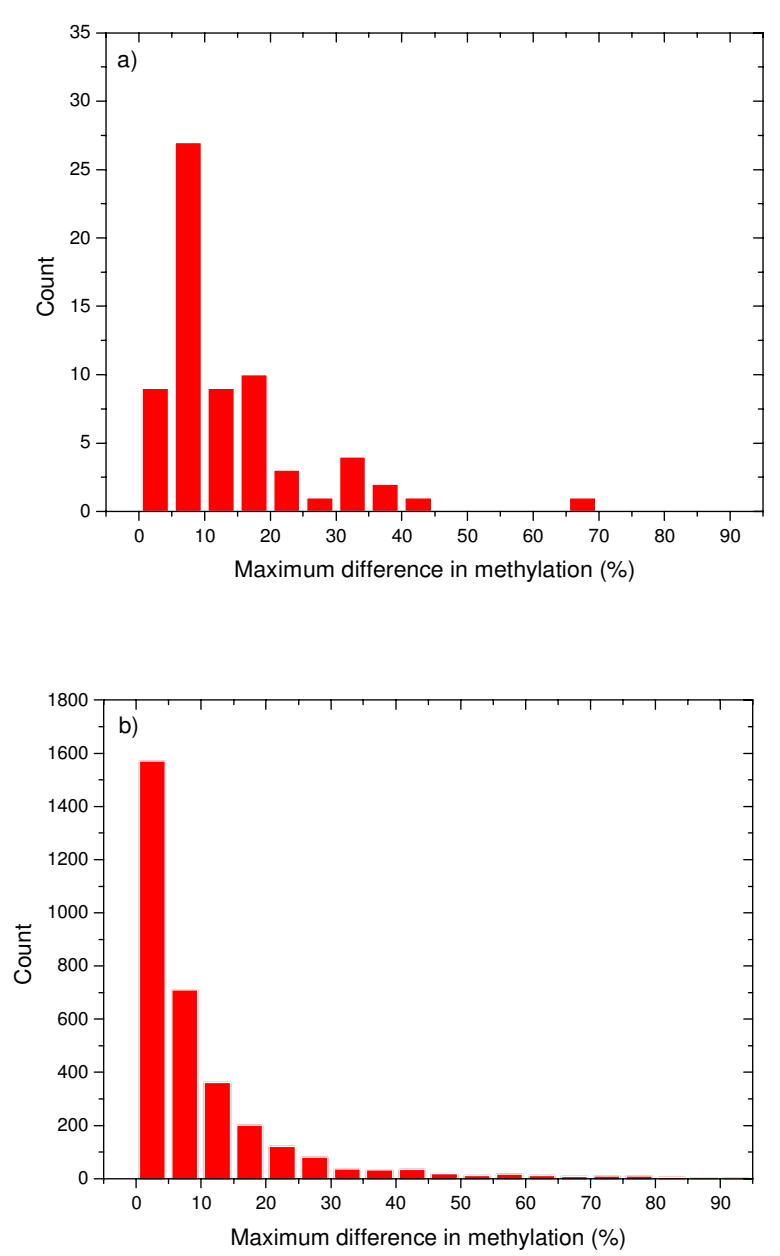

Figure 9 Distribution of the maximal methylation differences between CpGcluster islands within SWA (TJ) islands. a) HEP methylation data; b) Lister's methylation data [33].

positives. However, since CpGcluster [14] does not use any length threshold, it allows to identify short, but statistically significant $\mathrm{CpG}$ islets. A genome-wide search identifies a total of $88,137 \mathrm{CpG}$ islets in the human genome with $p$-value $\leq 10 \mathrm{E}-5$. Table 7 shows that relatively high percentages of $\mathrm{CpG}$ islets overlap with different sets of promoters and evolutionarily conserved elements, thus suggesting a functional role for many of the predicted islets. Noteworthy, a high proportion of these overlapping islets are exclusively predicted by $C p G$ cluster, but not by any of the remaining finders. This indicates that: 1) many of the small islands predicted by CpGcluster are not fragments of conventional islands, and 2) given the co-localization with functional regions, the islets might be indeed functional.

Using HEP data $[41,42]$ and Lister et al. methylation levels of single cytosines [33], we also determined the number of unmethylated and differentially methylated
CpG 'islets' (Table 8). A high proportion of the sampled CpG islets were unmethylated or differentially methylated, thus again suggesting a functional role for $\mathrm{CpG}$ islets. This is a very important point, as differential methylation of islands/islets may be involved in the regulation of gene expression. Again, the proportion of these $\mathrm{CpG}$ islets exclusively predicted by $C p G$ cluster is very high.

\section{Conclusions}

We systematically compared conventional SWA for detecting CGIs to a clustering method, namely the $C p G$ cluster algorithm. We showed than both approaches perform very similar when predicting long, unmethylated regions or polycomb sites. However, we found three scenarios where the CpGcluster algorithm seems to have advantages. First, the statistical significance assigned to each CpGcluster island seems to be a better quality parameter than the window size of conventional finders, as it reduces more efficiently false positive predictions. Second, we have shown that CpGcluster islands co-localize in a more specific way to alternative TSSs and methylation domains. Third, we have shown that many of the small islands predicted by CpGcluster might be functional, given the overlap with conserved elements or promoter regions. Moreover, $30 \%$ of the differentially methylated islets are exclusively predicted by $C p$ Gcluster, which suggests this method as the option of choice for the experimental verification of islet functionality.

\section{Methods}

\section{Sequence Data}

We used human genome assembly NCBI 36.1 (hg18), downloaded from the UCSC genome browser http:// hgdownload.cse.ucsc.edu/downloads.html\#human.

\section{Promoter data}

To quantify the co-localization of the predictions with promoter regions and principal transcription start sites we used the RefSeq gene annotation [43]. We furthermore used the DBTSS database version 6.0 [44], as it annotates also alternative transcription start sites, as well as start sites which cannot be assigned to a known RefSeq transcript. From both, the RefSeq and DBTSS annotation, we extracted the coordinates of two regions; the transcription start site (TSS) and the promoter regions, defined as TSS-1500 bp to TSS+500 bp.

\section{Genomic elements}

We determined the overlap of CGIs with conserved elements (PhastCons) and spurious Alu elements. The evolutionarily conserved elements [23] and the RepeatMasker [45] annotation of repeated elements where downloaded from the UCSC table browser [46]. In general, we consider two measures to quantify the over- 
Table 7: Overlap of CpG islets $(\mathrm{N}=\mathbf{8 8 , 1 3 7 )}$ with different sets of promoters and evolutionarily conserved elements.

\begin{tabular}{lcc}
\hline Genome element & Number of overlapping CpG islets & $\begin{array}{c}\text { Number of overlapping CpG islets } \\
\text { exclusively predicted by CpG cluster }\end{array}$ \\
\hline Promoters from RefSeq database & $9,826(11.15 \%)$ & $1,218(12.40 \%)$ \\
\hline TSSs from DBTSS database & $1,868(2.12 \%)$ & $398(21.31 \%)$ \\
\hline Promoter regions from DBTSS database & $6,510(7.39 \%)$ & $4,869(74.79 \%)$ \\
\hline PhastCons & & $8,219(46.66 \%)$ \\
\hline
\end{tabular}

lap between CGIs and genomic elements. First, we define the mean coverage of a CGI prediction as the mean value of all coverage fractions. The coverage fraction can be calculated as the number of bases of an island corresponding to a given genomic element divided by the island length. Furthermore, we calculate the overlap fraction as the number of islands which overlap in at least one base with a given genomic element divided by the total number of predicted islands.

\section{Island predictions}

For SWA CGI finders, a CpG island was at least $200 \mathrm{bp}$ long, which excluded the detection of any shorter tracts. To detect CpG-rich regions, disregarding its length, we used a recently published $\mathrm{CpG}$ island finder algorithm (CpGcluster, [14]) which does not rely on any length threshold but directly predicts statistically significant CpG clusters. Briefly, the CpGcluster algorithm can be divided into two steps. First, based on a distance threshold, the individual CpGs which are below this threshold are clustered along the DNA sequence. Second, by means of the negative binomial distribution a $p$-value is assigned to each CpG cluster, which allows the prediction of highly significant clusters such as CpG islands.

We considered five computational predictions of $\mathrm{CpG}$ islands. For the $C p G$ cluster algorithm [14], we generated two prediction sets by setting the assigned $p$-value to two different thresholds. We generated a relaxed set with $p$ value $<=1 \mathrm{E}-5$ and a strict set by setting the threshold to 1E-20. We implemented the TJ algorithm, as explained in [21], by setting the thresholds to: length $\geq 500 \mathrm{bp}, \mathrm{GC}$ content $\geq 55 \%, \mathrm{Obs}_{\mathrm{CpG}} / \operatorname{Exp}_{\mathrm{CpG}} \geq 0.65$ and $\operatorname{minCpG}>=$ $0.6 * \mathrm{~L}_{\text {island }} / 16$ (to avoid "mathematical" islands). We generated the CpGproD prediction [38] running the program http://pbil.univ-lyon1.fr/software/cpgprod.html with default parameters. Finally, we downloaded the UCSC $\mathrm{CpG}$ island predictions from the UCSC table browser [46].

\section{Gene coexpression analysis}

We used the GeneAtlas2 expression data [32] to determine the co-expression of gene pairs sharing a bi-direc-

Table 8: Number of unmethylated and differentially methylated CpG 'islets'.

\begin{tabular}{|c|c|c|c|}
\hline Dataset & Methylation state* & Number of CpG islets & $\begin{array}{l}\text { CpG 'islets' exclusively } \\
\text { predicted by CpGcluster }\end{array}$ \\
\hline \multirow[t]{2}{*}{ HEP (12 tissues) $)^{* *}$} & Unmethylated & 126 & 1 \\
\hline & Differentially methylated & 26 & 8 \\
\hline \multirow[t]{2}{*}{ Lister et al. 2009 (2 cell lines) ${ }^{* * *}$} & Unmethylated & 4,460 & 1,472 \\
\hline & Differentially methylated & 373 & 295 \\
\hline \multicolumn{4}{|c|}{$\begin{array}{l}\text { *Unmethylated: average methylation } \leq 0.2 ; \text { differentially methylated: average methylation }<=0.2 \text { in at least one tissue } \& \text { average methylation } \\
>=0.8 \text { in at least one other tissue. } \\
\text { **The methylation state of } 246 \text { CpG 'islets' from chromosomes } 6,20 \text { and } 22 \text { was determined by using } 3,168 \text { individual CpG sites (HEP project). } \\
\text { We only included CpGs which have been detected in at least } 2 \text { clones or in at least } 6 \text { different tissues. } \\
\text { ***We used the sequence reads obtained by MethylC-Seq for two human cell lines [33], H1 human embryonic stem cells and IMR90 fetal lung } \\
\text { fibroblasts, to get the average methylation level of single cytosines at both DNA strands for these two methylomes. All islands need more } \\
\text { than } 50 \% \text { of its CpGs covered. Only cytosines covered by at least } 10 \text { reads were counted. }\end{array}$} \\
\hline
\end{tabular}


tional promoter. The "coexpression value" for a couple of genes is the ratio of the number of tissues in which both genes are simultaneously expressed (signal levels $>200$ ) or simultaneously not expressed (signal levels $<=200$ ), and the number of healthy tissues with expression data.

\section{Methylation data}

Since the lack of methylation of a CpG island is a very good indicator of function [25], we used several different sources of experimental methylation data. Weber et al. [26] detected methylation states in two different tissues, fibroblast and sperm. We extracted 13,277 non-overlapping regions which are unmethylated in at least one of the two tissues (scaled $5 \mathrm{mC} \log 2$ ratio $<0.3$ ). Next, we used $17,383 \mathrm{CpG}$ island recently detected in blood cells by means of a new technique [28].

Finally, we assigned methylation states (unmethylated, methylated and differentially methylated) to our $C p G$ cluster predictions by means of the data from the HEPhuman epigenome project [42]. The data comprises about 1.9 million CpG methylation values, obtained from the analysis of 2,524 amplicons across chromosomes 6, 20 and 22 in 43 samples (derived from 12 different tissues). We first calculated the mean methylation of each CpG dinucleotide over the different clones, then deleting all CpGs which have been detected in less than 2 clones or in less than 6 different tissues. Subsequently, the individual CpGs were labeled as methylated (mean methylation $>=$ 80 ), intermediate methylated (80-20) and unmethylated (under 20) for each of the different tissues. Next, we define the methylation states of the CpGs over the different tissues in the following way: i) methylated CpG: methylated in more than $50 \%$ of tissues and never unmethylated, ii) unmethylated CpG: unmethylated in more than $50 \%$ of tissues and never methylated, iii) differentially methylated CpG: both, methylated and unmethylated in different tissues, the number of intermediate methylation states being smaller than $50 \%$. Finally, we assign a methylation label to the CpG islands which have methylation data for more than 50\% of its CpGs: i) methylated: more than $50 \%$ of the CpGs are methylated and no unmethylated $\mathrm{CpG}$ exist, ii) unmethylated: more than $50 \%$ of the CpGs are unmethylated and no methylated CpG exist, iii) differentially methylated: more than $50 \%$ of all CpGs need to be differentially methylated.

We also used the sequence reads obtained by MethylCSeq for two human cell lines [33], H1 human embryonic stem cells and IMR90 fetal lung fibroblasts, to get the average methylation level of single cytosines at both DNA strands for these two methylomes. All islands need more than $50 \%$ of its CpGs covered. Only cytosines covered by at least 10 reads were counted.

\section{Assessing prediction quality}

When comparing the prediction of $\mathrm{CpG}$ islands to a gold standard (e.g. experimentally verified islands), we define:

- True Positives (TP): An island overlapping in at least $1 \mathrm{bp}$ with the gold standard

- False Positives (FP): An island not overlapping with the gold standard

- False Negative (FN): An island in the gold standard that has not been predicted.

By means of these values, we then calculate the sensitivity and the Positive Predictive Value (also known as specificity in the gene prediction field [27]):

$$
\begin{aligned}
& S_{n}=\frac{T P}{T P+F N} \\
& P P V^{L B}=\frac{T P}{T P+F P}
\end{aligned}
$$

Note that we consider all islands not overlapping with the gold standard as false positive predictions. However, no complete gold standard exists, and therefore an unknown number of these islands will be actually true positive predictions. This assumption does not affect the sensitivity, as FP does not occur in the equation, but it affects the PPV. Consequently, and since the PPV can only increase when some FPs turn out to be TPs, the value used in this work is the lower boundary PPV of the prediction, e.g. the worst case scenario when all islands which do not overlap with the gold standard are indeed false positives.

\section{List of abbreviations}

CGI: CpG island; CpG O/E ratio: Ratio between observed and expected CpG frequencies; CpG: dinucleotide CG; G $+\mathrm{C}$ content, $\% \mathrm{G}+\mathrm{C}$ : Molecular fraction of guanine and cytosine; PhastCons: Phylogenetic Conserved Elements; Sn: The sensitivity of the prediction; PPV: Positive Predictive Value of the prediction; SWA: Sliding-window approaches; TJ: Takai/Jones program or island; TSS: Transcription Start Site

\section{Competing interests}

The authors declare that they have no competing interests.

\section{Authors' contributions}

$\mathrm{MH}$ designed and performed the experiments and wrote the manuscript (with $J L O), G B$ performed the search for alternative promoters and differential methylation, PC and PLE carried out the theoretical analysis of CpG clustering and help with the interpretation of statistical results, CP determined the overlap of the CGIs predicted by different finders with the domains bound by polycomb repressive complex, and JLO designed the experiments and wrote the manuscript (with $\mathrm{MH}$ ). All the authors critically read and approved the final version.

\section{Acknowledgements}

We thank Andy Choo and Nicholas Wong, from the University of Melbourne (Parkville, Victoria, Australia), by sharing with us their data on CpG islets. We acknowledge the Spanish Government (Grant No. BIO2008-01353) and the Spanish Junta de Andalucía (Grant Nos. P06-FQM1858 and P07-FQM3163) financial support. 
MH acknowledges financial support from the 'Juan de la Cierva' grant from the Spanish Government. GB acknowledges financial support from the 'Programa de formación de investigadores del Departamento de Educación, Universidades e Investigación' grant from the Basque Country Government.

\section{Author Details}

1Dpto. de Genética, Facultad de Ciencias, Universidad de Granada, Campus de Fuentenueva s/n, 18071, Granada, Spain, 2Lab. de Bioinformática, Centro de Investigación Biomédica, PTS, Avda. del Conocimiento s/n, 18100, Granada, Spain, ${ }^{3}$ Dpto. de Física Aplicada II, E.T.S.I. de Telecomunicación, Universidad de Málaga 29071-Malaga, Spain, ${ }^{4}$ Division of Sleep Medicine, Brigham and Woman's Hospital, Harvard Medical School, Boston, MA 02115, USA, 5Dpto. de Ingeniería Mecánica y Minera, EPS Jaén-Universidad de Jaén, Campus Las Lagunillas s/n A3-008, 23071-Jaén, Spain and ${ }^{6}$ Computational Biology Unit, Bergen Center for Computational Science \& Sars Centre for Marine Molecular Biology, University of Bergen, Thormøhlensgt 55, 5008 Bergen, Norway

Received: 26 January 2010 Accepted: 26 May 2010

Published: 26 May 2010

\section{References}

1. Gardiner-Garden M, Frommer M: CpG islands in vertebrate genomes. Journal of molecular biology 1987, 196(2):261-282.

2. Larsen F, Gundersen G, Lopez R, Prydz H: CpG islands as gene markers in the human genome. Genomics 1992, 13(4):1095-1107.

3. Antequera F: Structure, function and evolution of $\mathrm{CpG}$ island promoters. Cell Mol Life Sci 2003, 60(8):1647-1658.

4. Bird A: DNA methylation patterns and epigenetic memory. Genes \& development 2002, 16(1):6-21.

5. Bird AP: CpG-rich islands and the function of DNA methylation. Nature 1986, 321(6067):209-213.

6. Zhu J, He F, Hu S, Yu J: On the nature of human housekeeping genes. Trends Genet 2008, 24(10):481-484.

7. Baylin SB, Esteller M, Rountree MR, Bachman KE, Schuebel K, Herman JG: Aberrant patterns of DNA methylation, chromatin formation and gene expression in cancer. Human molecular genetics 2001, 10(7):687-692.

8. De Smet C, Lurquin C, Lethe B, Martelange V, Boon T: DNA methylation is the primary silencing mechanism for a set of germ line- and tumorspecific genes with a CpG-rich promoter. Molecular and cellular biology 1999, 19(11):7327-7335

9. Esteller M, Corn PG, Baylin SB, Herman JG: A gene hypermethylation profile of human cancer. Cancer research 2001, 61(8):3225-3229.

10. Issa JP: CpG island methylator phenotype in cancer. Nature reviews 2004, 4(12):988-993.

11. Riazalhosseini $Y$, Hoheisel JD: Do we use the appropriate controls for the identification of informative methylation markers for early cancer detection? Genome biology 2008, 9(11):405.

12. Song F, Smith JF, Kimura MT, Morrow AD, Matsuyama T, Nagase H, Held WA: Association of tissue-specific differentially methylated regions (TDMs) with differential gene expression. Proceedings of the National Academy of Sciences of the United States of America 2005 102(9):3336-3341

13. Hsieh F, Chen SC, Pollard K: A Nearly Exhaustive Search for CpG Islands on Whole Chromosomes. In The International Journal of Biostatistics Volume 5. The Berkeley Electronic Press; 2009:1-24.

14. Hackenberg M, Previti C, Luque-Escamilla PL, Carpena P, Martínez-Aroza J, Oliver JL: CpGcluster: A distance-based algorithm for CpG-island detection. BMC Bioinformatics 2006, 7(1):446.

15. Glass JL, Thompson RF, Khulan B, Figueroa ME, Olivier EN, Oakley EJ, Van Zant G, Bouhassira EE, Melnick A, Golden A, et al.: CG dinucleotide clustering is a species-specific property of the genome. Nucleic acids research 2007, 35(20):6798-6807.

16. Sujuan $Y$, Asaithambi A, Liu Y: CpGIF: an algorithm for the identification of CpG islands. Bioinformation 2008, 2(8):335-338.

17. Irizarry RA, Wu H, Feinberg AP: A species-generalized probabilistic model-based definition of CpG islands. Mamm Genome 2009, 20(910):674-680.

18. Wu H, Caffo B, Jaffee HA, Irizarry RA, Feinberg AP: Redefining CpG islands using hidden Markov models. Biostatistics 2010 in press.

19. Hughes A, Rando OJ: Chromatin 'programming' by sequence - is there more to the nucleosome code than \%GC? J Bio/ 2009, 8(11):96.
20. Bock C, Walter J, Paulsen M, Lengauer T: CpG island mapping by epigenome prediction. PLoS Comput Biol 2007, 3(6):e110.

21. Takai $D$, Jones PA: Comprehensive analysis of $C p G$ islands in human chromosomes 21 and 22. Proceedings of the National Academy of Sciences of the United States of America 2002, 99(6):3740-3745.

22. Han L, Zhao Z: CpG islands or CpG clusters: how to identify functional GC-rich regions in a genome? BMC Bioinformatics 2009, 10:65.

23. Siepel A, Bejerano G, Pedersen JS, Hinrichs AS, Hou M, Rosenbloom K, Clawson H, Spieth J, Hillier LW, Richards S, et al:: Evolutionarily conserved elements in vertebrate, insect, worm, and yeast genomes. Genome Res 2005, 15(8):1034-1050.

24. Saxonov S, Berg P, Brutlag DL: A genome-wide analysis of CpG dinucleotides in the human genome distinguishes two distinct classes of promoters. Proceedings of the National Academy of Sciences of the United States of America 2006, 103(5):1412-1417.

25. Illingworth RS, Bird AP: CpG islands--'a rough guide'. FEBS letters 2009, 583(11):1713-1720.

26. Weber M, Hellmann I, Stadler MB, Ramos L, Paabo S, Rebhan M, Schubeler $D$ : Distribution, silencing potential and evolutionary impact of promoter DNA methylation in the human genome. Nature genetics 2007, 39(4):457-466

27. Burset M, Guigo R: Evaluation of gene structure prediction programs. Genomics 1996, 34(3):353-367

28. Illingworth R, Kerr A, Desousa D, Jorgensen H, Ellis P, Stalker J, Jackson D, Clee C, Plumb R, Rogers J, et al:: A novel CpG island set identifies tissuespecific methylation at developmental gene loci. PLoS biology 2008, 6(1):e22.

29. Previti C, Harari O, Zwir I, del Val C: Profile analysis and prediction of tissue-specific CpG island methylation classes. BMC Bioinformatics 2009, 10:116.

30. Tanay A, O'Donnell AH, Damelin M, Bestor TH: Hyperconserved CpG domains underlie Polycomb-binding sites. Proceedings of the National Academy of Sciences of the United States of America 2007 104(13):5521-5526

31. Yamashita R, Suzuki Y, Wakaguri H, Tsuritani K, Nakai K, Sugano S: DBTSS: DataBase of Human Transcription Start Sites, progress report 2006. Nucleic acids research 2006:D86-89.

32. Su Al, Wiltshire T, Batalov S, Lapp H, Ching KA, Block D, Zhang J, Soden R, Hayakawa M, Kreiman G, et al:: A gene atlas of the mouse and human protein-encoding transcriptomes. Proceedings of the National Academy of Sciences of the United States of America 2004, 101(16):6062-6067.

33. Lister R, Pelizzola M, Dowen RH, Hawkins RD, Hon G, Tonti-Filippini J, Nery JR, Lee L, Ye Z, Ngo QM, et al:: Human DNA methylomes at base resolution show widespread epigenomic differences. Nature 2009, 462(7271):315-322

34. Hodges E, Smith AD, Kendall J, Xuan Z, Ravi K, Rooks M, Zhang MQ, Ye K, Bhattacharjee $A$, Brizuela $L$, et al:: High definition profiling of mammalian DNA methylation by array capture and single molecule bisulfite sequencing. Genome Res 2009, 19(9):1593-1605.

35. Wong NC, Wong LH, Quach JM, Canham P, Craig JM, Song JZ, Clark SJ, Choo KH: Permissive transcriptional activity at the centromere through pockets of DNA hypomethylation. PLoS genetics 2006, 2(2):e17.

36. Li W, Bernaola-Galván P, Haghighi F, Grosse I: Applications of recursive segmentation to the analysis of DNA sequences. Computers \& chemistry 2002, 26(5):491-510.

37. Luque-Escamilla PL, Martínez-Aroza J, Oliver JL, Gómez-Lopera JF, RománRoldán R: Compositional searching of $\mathrm{CpG}$ islands in the human genome. Phys Rev E 2005, 71:6.

38. Ponger L, Mouchiroud D: CpGProD: identifying CpG islands associated with transcription start sites in large genomic mammalian sequences. Bioinformatics (Oxford, England) 2002, 18(4):631-633.

39. Takai $D$, Jones PA: The $C p G$ island searcher: a new WWW resource. silico biology 2003, 3(3):235-240.

40. Wang Y, Leung FC: An evaluation of new criteria for CpG islands in the human genome as gene markers. Bioinformatics (Oxford, England) 2004, 20(7):1170-1177.

41. Human Epigenome Project [http://www.epigenome.org/

42. Eckhardt F, Lewin J, Cortese R, Rakyan VK, Attwood J, Burger M, Burton J, Cox TV, Davies R, Down TA, et al: DNA methylation profiling of human chromosomes 6, 20 and 22. Nature genetics 2006, 38(12):1378-1385. 
43. Pruitt KD, Tatusova T, Maglott DR: NCBI reference sequences (RefSeq): a curated non-redundant sequence database of genomes, transcripts and proteins. Nucleic acids research 2007:D61-65.

44. Wakaguri H, Yamashita R, Suzuki Y, Sugano S, Nakai K: DBTSS: database of transcription start sites, progress report 2008. Nucleic acids research 2008:D97-101.

45. RepeatMasker [http://www.repeatmasker.org/

46. Karolchik D, Kuhn RM, Baertsch R, Barber GP, Clawson H, Diekhans M, Giardine B, Harte RA, Hinrichs AS, Hsu F, et al.: The UCSC Genome Browser Database: 2008 update. Nucleic acids research 2008:D773-779.

doi: 10.1186/1471-2164-11-327

Cite this article as: Hackenberg et al., Prediction of CpG-island function: CpG clustering vs. sliding-window methods BMC Genomics 2010, 11:327

Submit your next manuscript to BioMed Centra and take full advantage of:

- Convenient online submission

- Thorough peer review

- No space constraints or color figure charges

- Immediate publication on acceptance

- Inclusion in PubMed, CAS, Scopus and Google Scholar

- Research which is freely available for redistribution

Submit your manuscript at www.biomedcentral.com/submit 